\title{
Modelling the interaction between flooding events and economic growth
}

\author{
J. Grames ${ }^{1}$, A. Prskawetz ${ }^{2}$, D. Grass ${ }^{3}$, and G. Blöschl ${ }^{4}$ \\ ${ }^{1}$ Centre for Water Resource Systems, Vienna University of Technology, Vienna, Austria \\ ${ }^{2}$ Institute of Statistics and Mathematical Methods in Economics, Research Unit Economics, \\ Vienna University of Technology, Vienna, Austria \\ ${ }^{3}$ Institute of Statistics and Mathematical Methods in Economics, Research Unit Operations Research and \\ Control Systems, Vienna University of Technology, Vienna, Austria \\ ${ }^{4}$ Institute of Hydrologic Engineering and Water Resources Management, Vienna University of Technology, \\ Vienna, Austria \\ Correspondence to: J. Grames (grames@waterresources.at)
}

Received: 14 April 2015 - Accepted: 14 April 2015 - Published: 11 June 2015

\begin{abstract}
Socio-hydrology describes the interaction between the socio-economy and water. Recent models analyze the interplay of community risk-coping culture, flooding damage and economic growth (Di Baldassarre et al., 2013; Viglione et al., 2014). These models descriptively explain the feedbacks between socio-economic development and natural disasters like floods. Contrary to these descriptive models, our approach develops an optimization model, where the intertemporal decision of an economic agent interacts with the hydrological system. In order to build this first economic growth model describing the interaction between the consumption and investment decisions of an economic agent and the occurrence of flooding events, we transform an existing descriptive stochastic model into an optimal deterministic model. The intermediate step is to formulate and simulate a descriptive deterministic model. We develop a periodic water function to approximate the former discrete stochastic time series of rainfall events.

Due to the non-autonomous exogenous periodic rainfall function the long-term path of consumption and investment will be periodic.
\end{abstract}

\section{Introduction}

During the past decades more and more floods have occurred, especially in central and eastern Europe. Still, people like to settle close to rivers to reap economic advantages: rivers enable ways of transport, supply water for industry and agriculture and enhance the quality of living due to lively nature and beautiful scenery. But the awareness of flooding has increased, and therefore societies develop projects like building levees to avoid or at least reduce flood damage. These investments are costly, but avoid damage in the future. Simulation models can describe this interesting trade-off structure. But analysing the investment strategies is even more meaningful in an economic decision framework. Consequently, we de- velop an optimization model based on existing descriptive socio-hydrology models.

Viglione et al. (2014) and Di Baldassarre et al. (2013) developed such models to explain the feedbacks between settlements close to rivers and flooding events. Protecting a settlement can, however, increase the damage of the settlement downwards the river. Furthermore, higher levees or any other defence capital are lowering the risk of floods and therefore may even further increase the willingness to settle close to the river. But if water levels rise higher than the levees, the physical capital next to the river is destroyed. Since there is a higher physical capital stock next to the river, the flood hits the economy even harder.

The model of Viglione describes the dynamics of four key variables: the size of the human settlement $G(t)$ (number of 
people, physical size of settlement, economic wealth), the distance between the settlement and the river $D(t)$, the height of the levees $H(t)$ and the memory of flooding events $M(t)$. We present a short summary of the model in the following as it will serve as the benchmark model for our alternative set up of a deterministic model. Note, Greek letters are exogenous parameters and capital letters are endogenous variables described by the model.

The flood intensity

$F(t)= \begin{cases}1-\exp \left(-\frac{W(t)+\xi_{\mathrm{H}} H_{-}}{\alpha_{\mathrm{H}} D(t)}\right) & \text { if } W(t)+\xi_{\mathrm{H}} H_{-}>H_{-} \\ 0 & \text { else }\end{cases}$

is bounded by the interval $[0,1]$ and measures the relative damage of the economic wealth in case of a flood. A flood occurs if the effective water level $W(t)+\xi_{\mathrm{H}} H_{-}$exceeds the height of the levees $H_{-}$. The higher the effective water level and the closer to the river the settlement is located (i.e. the higher the value of $D$ ), the higher is the flood intensity.

People raise the levees by the amount $R(t)=\varepsilon_{T}\left(W(t)+\xi_{\mathrm{H}} H_{-}-H_{-}\right), \quad$ if a flood occurs $(F(t)>0)$, the flood damage $\left(F(t) G_{-}\right)$is higher than the costs of raising the levees $\left(\gamma_{\mathrm{E}} R(t) \sqrt{G_{-}}\right)$ and the economy can still effort to build them $\left(G_{-}-F(t) G(t)>\gamma_{\mathrm{E}} R(t) \sqrt{G_{-}}\right)$. Raising the levees yields a lower shock magnitude $\left(S=\alpha_{\mathrm{S}} F(t)\right)$ than without $(S=F(t))$. The dynamics are described by the four key variables mentioned above:

$$
\begin{aligned}
\frac{\mathrm{d} G}{\mathrm{~d} t} & =\phi_{\mathrm{E}}(1-D(t)) G(t)-\Delta(\Upsilon(t))\left[F(t) G(t)+\gamma_{\mathrm{E}} R(t) \sqrt{G(t)}\right] \\
\frac{\mathrm{d} D}{\mathrm{~d} t} & =\left(M-\frac{D}{\lambda_{\mathrm{P}}}\right) \frac{\varphi_{\mathrm{P}}}{\sqrt{G(t)}} \\
\frac{\mathrm{d} H}{\mathrm{~d} t} & =\Delta(\Upsilon(t)) R(t)-\kappa_{\mathrm{T}} H \\
\frac{\mathrm{d} M}{\mathrm{~d} t} & =\Delta(\Upsilon(t)) S(t)-\mu_{\mathrm{S}} M(t)
\end{aligned}
$$

$\Delta(\Upsilon(t))$ equals one if a flooding event occurs and zero the rest of the time. The change in economic wealth $G(t)$ is decreasing if the settlement lives further away from the river $(D(t))$ and if a flood hits (see Eq. 2). In order to increase the economic wealth, people tend to move closer to the river unless the memory of the last flood is still high (Eq. 3).

Equation (4) assumes that levees depreciate over time except in those periods where people are raising them due to the occurrence of flooding events. Similar, the memory of people about flooding events depreciates over time if no shock appears due to a new flood (Eq. 5).

In the model (Viglione et al., 2014) described by Eqs. (1) to (5) the timing and the height of the flooding events are specified with a stochastic function. In a first step we approximate the stochastic water level by a deterministic periodic function. We next set up an optimal dynamic control model that allows for the optimal decision of the amount to be invested into defence capital that protects societies from flooding. The objective to be maximized is a societal welfare function that depends on consumption which in turn depends on the investment into productive capital. Hence, we are faced with an intertemporal optimal investment decision between defence versus productive capital.

\section{Introducing optimization into socio-hydrology}

Dynamic optimization methods are rarely used in sociohydrology. In the nineties Sritharan (1992) used optimal control in hydrodynamics and flow analysis, but did not capture the socio-economic perspective. Chahim et al. (2013) were the first who used optimal control to derive the optimal timing of dike heightenings as well as the corresponding optimal dike heightenings to protect against floods.

The aim of dynamic optimization is to control a dynamic system such as mechanical motions, physical processes, and economic systems in an optimal way. Optimization is not only a goal, it could be a tool for understanding the mechanisms of a system (Veliov, 2012). We can use both analytical and numerical methods to solve optimization problems. In this paper we focus on analytical solutions, but also consider numerical methods once analytical solutions are no longer feasible.

An optimization problem consists of an objective function that is maximized or minimized with respect to control variables, and subject to the dynamics of the variables involved (termed state equations) and possibly additional constraints on control and state variables. The Lagrangian method is used to derive optimal static solutions. In contrast dynamic optimization problems are more complicated as they involve intertemporal decisions (e.g. investing today or later) that in turn determine the evolution of state variables (e.g. capital) and hence the dynamic evolution of the objective function. We can apply optimal control theory or dynamic programming to solve dynamic optimization problems. For analytical solutions it is more popular to use optimal control theory. The main methods are the use of the Pontryagin's maximum principle (Pontryagin, 1962) and the Hamilton-Jacobi-Bellman equation (Bellman, 1954). In the following we only refer to deterministic optimal control theory.

\subsection{Requirements for optimization}

In order to set up a deterministic optimal control model we first approximate the water level function by a deterministic periodic function and secondly we transform all discontinuous functions to continuously differentiable functions.

\subsection{The continuous water function}

Rainfall is part of the water cycle and appears in a more or less regular way. We therefore apply a periodic function to model the water level after such rainfall events (Yevjevich and Harmaciocjlu, 1990; Zakaria, 2001). Moreover, we also 
a)

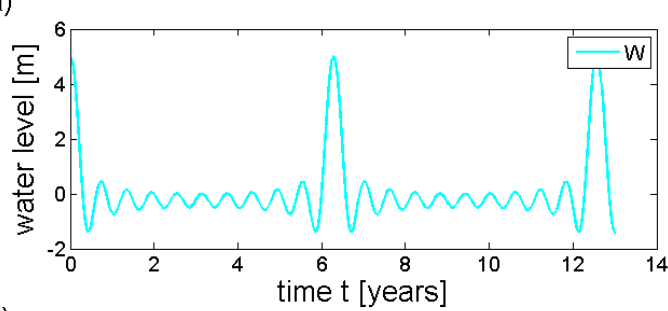

b)

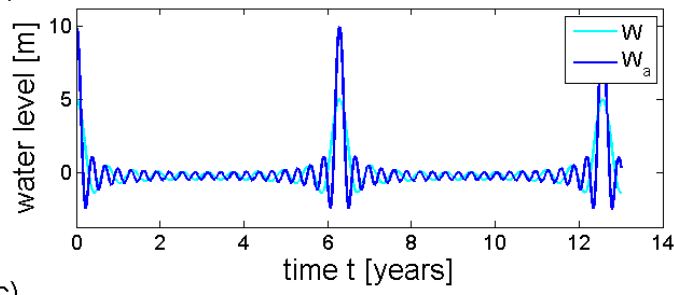

c)

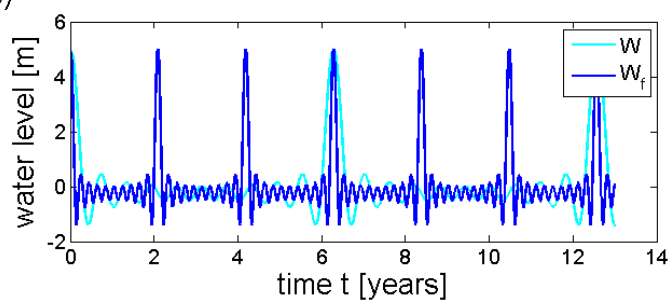

Figure 1. (a) The periodic continuous water function. (b) The water function $W_{\mathrm{a}}$ with a higher amplitude compared to the initial water function $W$. (c) The water function $W_{\mathrm{f}}$ with a higher frequency compared to the initial water function $W$.

have to represent extreme rain events. Obviously, such extreme events do not occur as often as normal rainfall events. Together with Langer (2014) we developed the following periodic function to approximate the periodic water level.

$W(t)=\frac{1}{2} \sum_{\kappa_{i}=1}^{\kappa_{s}} \cos \left(\kappa_{m} \kappa_{i} t\right)$

Figure 1a shows two periods of this function using $\kappa_{s}=10$ and $\kappa_{m}=1$. Of course, we can change the amplitude of the water level and the frequency of the extreme rainfall events choosing the parameter $\kappa_{s}$ and $\kappa_{m}$, respectively. Figure $1 \mathrm{~b}$ displays function (Eq. 6) for $\kappa_{s}=20$ and $\kappa_{m}=1$ and Fig. 1c indicates a higher frequency using $\kappa_{s}=10$ and $\kappa_{m}=3$.

Since water levels can only be positive we transform the water level in Eq. (6) to the positive water level function:

$W(t)=\max \left[0, \frac{1}{2} \sum_{\kappa_{i}=1}^{\kappa_{s}} \cos \left(\kappa_{m} \kappa_{i} t\right)\right]$.

Note, function (Eq. 7) is not continuous differentiable. In case we do not set the basic water level zero but want $W(t)=0$ to represent dry times we can shift the function (Eq. 6) and write

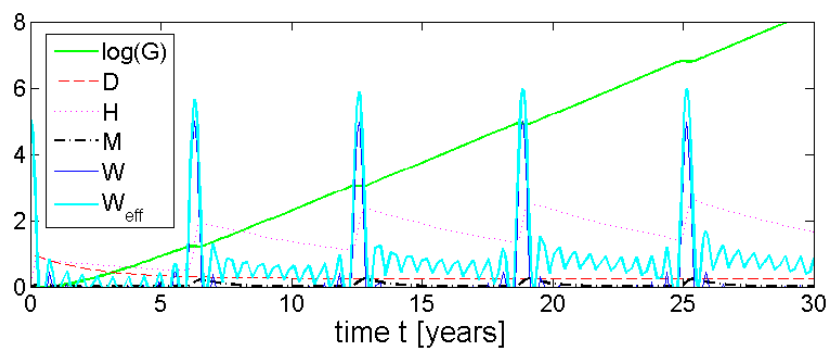

Figure 2. A simulation of the key dynamics of Viglione et al. (2014) given the continuous water function. It shows economic wealth $G$, distance $D$, height of levees $H$, memory $M$, basic water level $W$ and effective water level $W_{\text {eff }}$.

$W(t)=\frac{1}{2} \sum_{\kappa_{i}=1}^{\kappa_{s}} \cos \left(\kappa_{m} \kappa_{i} t\right)+\zeta \mathrm{W}$

with the positive constant

$\zeta_{\mathrm{W}}=\mid \min _{t \in[0,2 \pi]}\left[\frac{1}{2} \sum_{\kappa_{i}=1}^{\kappa_{s}} \cos \left(\kappa_{m} \kappa_{i} t\right)\right]$.

This guarantees that the water level $W(t)$ is always larger than or equal to zero.

For the following analysis we use function (Eq. 6) in order to have a continuous and twice differentiable function.

\section{Results}

To demonstrate the effects and plausibility of the continuous water function we simulated the model of Viglione et al. (2014) using the continuous input function (Eq. 6) instead of the random rainfall events. Figure 2 shows the dynamics of the model based on the new water function. We also provided the simulation of the model in Fig. 3 using the stochastic rainfall events from Viglione et al. (2014).

The economy is growing exponentially, even though it decays during every flooding event. This is not only because of the damage that occurs, but also because of the investments in raising the levees. The levees can avoid small floods, but the frequency of the large floods is too low to sustain depreciation and protect the settlements with levees. However, in the first decade of the simulation the settlement moves closer to the river, but remains half way for the rest of the time. On the one hand, the memory is not significantly high over a longer period to make the settlement move further away. Every time a huge flood occurs, the memories are present, but people forget very fast and after around two years they do not care about past floods any more. On the other hand, the size of the settlement and the economy is already so extended that it is hard to move.

To sum up, both simulations in Figs. 2 and 3 show qualitatively similar results. Even though the timing and the duration of the high water levels due to extreme rainfall events are 


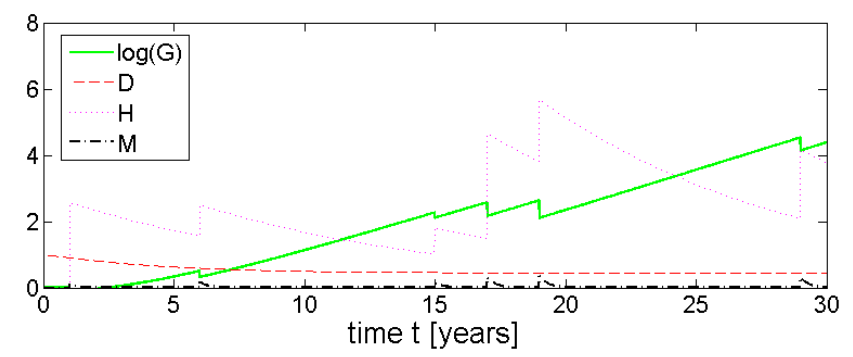

Figure 3. A simulation of the key dynamics of Viglione et al. (2014) using the original random rainfall events. It shows economic wealth $G$, distance $D$, height of levees $H$ and memory $M$.

slightly different, in the long term the consequences of floods lead to the same qualitative behaviour of the variables.

Specifically, we can identify a periodic behaviour of the system after some decades in Fig. 2, whereas the random events obviously cause rather unpredictable behaviour.

\section{Conclusion and outlook}

Our aim is to demonstrate how optimal control theory can be applied in socio-hydrology. In a first step we aim to apply a deterministic framework. For this purpose we have shown how an existing descriptive stochastic sociohydrology model can be transformed into a deterministic framework. For this purpose we substituted the exogenous function of rainfall by a periodic deterministic function. Applying the new water function yields results that can approximate the stochastic original model quite well.

The next step is to formulate an optimization model within an economic framework.
Acknowledgements. The authors would like acknowledge financial support from the Austrian Science Funds (FWF) as part of the Vienna Doctoral Programme on Water Resource Systems (DKplus W1219-N22) and thank their colleagues within the doctoral programme for continuing support and the discussions within the cluster meetings.

\section{References}

Bellman, R. E.: Dynamic programming and a new formalism in the calculus of variations, P. Natl. Acad. Sci. USA, 40, 231-235, 1954.

Chahim, M., Brekelmans, R. C. M., den Hertog, D., and Kort, P. M.: An impulse control approach to dike height optimization, Optimiz. Meth. Softw., 28, 458-477, 2013.

Di Baldassarre, G., Viglione, A., Carr, G., Kuil, L., Salinas, J. L., and Blöschl, G.: Socio-hydrology: conceptualising humanflood interactions, Hydrol. Earth Syst. Sci., 17, 3295-3303, doi:10.5194/hess-17-3295-2013, 2013.

Langer, S.: Sociohydrology: Conceptualising human-flood interactions, Diploma Thesis, Vienna University of Technology, Vienna, 2014.

Pontryagin, L. S.: Mathematical Theory of Optimal Processes, edited by: Pontryagin, L. S. and Boltyanskii, V. G., John Wiley \& Sons Inc, 1962.

Sritharan, S. S.: An optimal control problem in exterior hydrodynamics, P. Roy. Soc. Edinburgh, 121A, 5-32, 1992.

Veliov, V. M.: Dynamic Optimization, Course script, Vienna University of Technology, Vienna, 2012.

Viglione, A., Di Baldassarre, G. Brandimarte, L., Kuil, L., Carr, G., Salinas, J. L., and Bloeschl, G.: Insights from socio-hydrology modelling on dealing with flood risk - roles of collective memory, risk-taking attitude and trust, J. Hydrol., 518A, 71-82, doi:10.1016/j.jhydrol.2014.01.018, 2014.

Yevjevich, V. and Harmanciocjlu, N. B.: Periodic-Stochastic Modeling of Separation of Precipitation Into Rainfall and Snowfall, Water Resour. Res., 26, 2613-2623, 1990.

Zakaria, A.: A study of periodic and stochastic modeling of monthly rainfall from Purajaya station, Asian T. Eng., 1, 1-7, 2011. 